\title{
Hippocampal Theta Oscillations Support Successful Associative Memory Formation
}

\author{
${ }^{\circledR}$ Srinivas Kota, ${ }^{1}{ }^{\circledR}$ Michael D. Rugg, ${ }^{2}$ and Bradley C. Lega ${ }^{1}$ \\ ${ }^{1}$ Department of Neurosurgery, University of Texas Southwestern Medical Center, Dallas, Texas 75390 , and ${ }^{2}$ Center for Vital Longevity, University of \\ Texas at Dallas, Dallas, Texas 75390
}

Models of memory formation posit that episodic memory formation depends critically on the hippocampus, which binds features of an event to its context. For this reason, the contrast between study items that are later recollected with their associative pair versus those for which no association is made should reveal electrophysiological patterns in the hippocampus selectively involved in associative memory encoding. Extensive data from studies in rodents support a model in which theta oscillations fulfill this role, but results in humans have not been as clear. Here, we used an associative recognition memory procedure to identify hippocampal correlates of successful associative memory encoding and retrieval in patients (10 females and 9 males) undergoing intracranial EEG monitoring. We identified a dissociation between $2-5 \mathrm{~Hz}$ and $5-9 \mathrm{~Hz}$ theta oscillations, by which power increases in $2-5 \mathrm{~Hz}$ oscillations were uniquely linked with successful associative memory in both the anterior and posterior hippocampus. These oscillations exhibited a significant phase reset that also predicted successful associative encoding and distinguished recollected from nonrecollected items at retrieval, as well as contributing to relatively greater reinstatement of encoding-related patterns for recollected versus nonrecollected items. Our results provide direct electrophysiological evidence that $2-5 \mathrm{~Hz}$ hippocampal theta oscillations preferentially support the formation of associative memories, although we also observed memory-related effects in the $5-9 \mathrm{~Hz}$ frequency range using measures such as phase reset and reinstatement of oscillatory activity.

Key words: associative recognition; episodic memory; hippocampus; intracranial electroencephalography

Significance Statement

Models of episodic memory encoding predict that theta oscillations support the formation of interitem associations. We used an associative recognition task designed to elicit strong hippocampal activation to test this prediction in human neurosurgical patients implanted with intracranial electrodes. The findings suggest that $2-5 \mathrm{~Hz}$ theta oscillatory power and phase reset in the hippocampus are selectively associated with associative memory judgments. Furthermore, reinstatement of oscillatory patterns in the hippocampus was stronger for successful recollection. Collectively, the findings support a role for hippocampal theta oscillations in human associative memory.

\section{Introduction}

Models of episodic memory encoding posit that hippocampal theta oscillations fulfill the critical role of organizing neuronal ensembles to link together item and contextual representations (Staudigl and Hanslmayr, 2013; Eichenbaum, 2017). Numerous experimental findings have established the importance of theta oscillations for associative memory using spatial paradigms in rodents, including the observation that interventions that disrupt

\footnotetext{
Received Mar. 30, 2020; revised 0ct. 23, 2020; accepted 0ct. 28, 2020.

Author contributions: M.D.R. and B.C.L. designed research; S.K. and B.C.L. performed research; S.K. analyzed data; S.K., M.D.R., and B.C.L. wrote the paper.

This work was funded by National Institute of Neurological Disorders and Stroke-National Institutes of Health (Grant R01 NS107357 to B.C.L.) and the Texas Health Resources Clinical Scholars Program (to B.C.L.).

The authors declare no competing financial interests.

Correspondence should be addressed to Bradley C. Lega at Bradley.Lega@UTSouthwestern.edu.

https://doi.org/10.1523/JNEUROSCI.0767-20.2020

Copyright $\odot 2020$ the authors
}

theta oscillations attenuate associative encoding (Yamaguchi et al., 2007; Stella and Treves, 2011). However, the role of hippocampal theta oscillations in human associative memory has proven to be more difficult to establish. In spatial navigation and free recall, theta power increases in the $2-5 \mathrm{~Hz}$ frequency range have been reported to predict successful memory encoding, and additional properties such as phase amplitude coupling, phase reset, and phase synchronization have also been described for oscillations in this frequency range (Ekstrom et al., 2005; Lega et al., 2012; Lin et al., 2017). However, most studies examining hippocampal oscillatory changes during mnemonic processing report power decreases in the $2-10 \mathrm{~Hz}$ band (Greenberg et al., 2015; Fellner et al., 2016). By contrast, intracranial EEG (iEEG) studies using recognition memory paradigms report properties such as phase amplitude coupling, phase reset, and phase locking of single units that include faster theta frequencies up to $9 \mathrm{~Hz}$ (Axmacher et al., 2006; Fell and Axmacher, 2011). On the one 
Table 1. Demographic data including subject's age, duration of epilepsy, hemispheres of onset, number of electrodes in the anterior and posterior hypothalamus, and location of seizure onset

\begin{tabular}{|c|c|c|c|c|c|c|c|}
\hline $\begin{array}{l}\text { Subject } \\
\text { no. }\end{array}$ & Sex & $\begin{array}{l}\text { Age } \\
\text { (years) }\end{array}$ & $\begin{array}{l}\text { Duration of } \\
\text { epilepsy (years) }\end{array}$ & $\begin{array}{l}\text { Hemisphere } \\
\text { of onset }\end{array}$ & $\begin{array}{l}\text { Electrodes } \\
\text { in } \mathrm{AH}\end{array}$ & $\begin{array}{l}\text { Electrodes } \\
\text { in PH }\end{array}$ & Location of seizure onset \\
\hline 1 & $\mathrm{~F}$ & 38 & 14 & B & 6 & 5 & Right heterotopia, left heterotopia \\
\hline 2 & $\mathrm{~F}$ & 31 & 6 & $\mathrm{~L}$ & 0 & 1 & Left insula \\
\hline 3 & $\mathrm{~F}$ & 22 & 9 & $\mathrm{~L}$ & 4 & 1 & Left fusiform gyrus \\
\hline 4 & $\mathrm{~F}$ & 36 & 19 & $\mathrm{R}$ & 0 & 2 & Right anterior cingulate/right SFG prefrontal, right anterior insula/frontal operculum \\
\hline 5 & M & 27 & 21 & B & 5 & 2 & Bilateral hippocampi \\
\hline 6 & M & 41 & 30 & B & 4 & 0 & Amygdala, right insula \\
\hline 7 & M & 54 & 30 & $\mathrm{R}$ & 1 & 2 & Superior temporal sulcus/middle temporal gyrus posterior \\
\hline 8 & M & 26 & 2 & $\mathrm{R}$ & 5 & 2 & Right temporal lobe \\
\hline 9 & M & 22 & 3 & B & 3 & 3 & Bilateral temporal lobe \\
\hline 10 & $\mathrm{~F}$ & 42 & 8 & $\mathrm{~L}$ & 3 & 1 & Left posterior insula \\
\hline 11 & M & 31 & 27 & $\mathrm{R}$ & 3 & 0 & Right hippocampus \\
\hline 12 & $\mathrm{~F}$ & 54 & 4 & $\mathrm{~L}$ & 3 & 2 & Left entorhinal \\
\hline 13 & M & 20 & 4 & B & 5 & 4 & Right collateral sulcus/fusiform gyrus/ heterotopia \\
\hline 14 & $\mathrm{~F}$ & 37 & 4 & $\mathrm{~L}$ & 2 & 1 & Left temporal pole \\
\hline 15 & $\mathrm{~F}$ & 31 & 10 & $\mathrm{~L}$ & 6 & 6 & Left anterior hippocampus, amygdala \\
\hline 16 & $\mathrm{~F}$ & 22 & 17 & $\mathrm{~L}$ & 4 & 2 & Left hippocampus \\
\hline 17 & M & 23 & 6 & $\mathrm{R}$ & 6 & 0 & Right prefrontal/premotor \\
\hline 18 & M & 45 & 12 & $\mathrm{R}$ & 5 & 3 & Right hippocampus \\
\hline 19 & $\mathrm{~F}$ & 45 & 23 & $\mathrm{~L}$ & 6 & 2 & Left schizencephalic \\
\hline
\end{tabular}

L, Left hemisphere; $\mathrm{R}$, right hemisphere; F, female; $\mathrm{M}$, male; B, bilateral; $\mathrm{AH}$, anterior hippocampus; $\mathrm{PH}$, posterior hippocampus.

hand, these findings support the general importance of theta oscillations in human memory but observing these properties within a simple recognition paradigm (rather than an episodic or spatial navigation task) may not necessarily support a model by which theta oscillations specifically support item-context associations, as predicted from rodent data.

This question may in turn be related to possible differences between $2-5 \mathrm{~Hz}$ "slow" theta oscillations and $5-9 \mathrm{~Hz}$ "fast" theta oscillations in human iEEG recordings from the hippocampus (Jacobs, 2014). While many reports of hippocampal theta do not attempt to segregate oscillations within the broad $2-9 \mathrm{~Hz}$ frequency range, some human data suggest that it is oscillations $<5 \mathrm{~Hz}$ that exhibit mnemonic correlates consistent with predictions from rodent experiments, including power increases during successful, compared with unsuccessful, encoding and phase amplitude coupling (Ekstrom et al., 2005; Lega et al., 2012, 2016; Lin et al., 2017).

A central tenet of episodic memory retrieval is the concept of reinstatement, meaning that the brain patterns present at the time of encoding recur in the brain at the time of retrieval. Across both invasive and noninvasive modalities, evidence of memory reinstatement has been demonstrated in human studies (Thakral et al., 2015; Staresina et al., 2016). A clear prediction of these data related to the associative recognition task is that reinstatement (as quantified by distance metrics such as cosine similarity) should be greater for recollected items compared with nonrecalled items. Understanding how theta oscillations participate in reinstatement-related activity patterns would further clarify their role in human memory.

We used an associative recognition procedure previously validated in human fMRI studies (de Chastelaine et al., 2016a,b). A key feature of this procedure is that it permits a "process pure" contrast between patterns of brain activity linked with successful versus unsuccessful recollection, making it well suited for neurophysiological examinations of hippocampal processing supporting associative memory (compared with source memory tasks more generally). Our primary goal was to ascertain whether theta power would differ according to whether a study item went on to be successfully recollected (associative hit) or to be wrongly judged as rearranged (associative miss). Participants included 19 individuals in whom intracranial electrodes had been inserted into the hippocampus. This allowed us to interpret our findings in light of models of hippocampal longitudinal specialization (Poppenk et al., 2013).

\section{Materials and Methods}

Nineteen adult participants (age range $=20-54$ years; mean age $=$ 34 years, $\mathrm{SD}=11$ years; 10 females; 15 right-handed participants; Table 1) with pharmacologically resistant epilepsy were implanted with stereotactic EEG electrodes to detect seizure location. The number and location of the intracerebral electrodes implanted in the patients were determined exclusively based on the clinical need. The participants were recruited from the Epilepsy Monitoring Unit at the University of Texas Southwestern Medical Center (Dallas, TX). Each participant provided informed consent before participation in the research study in accordance with the University of Texas Southwestern Medical Center Institutional Review Board. Data collected from eight additional individuals were excluded because of an insufficient number of trials or inadequate behavioral performance, falling below an a priori defined cutoff of 0 for associative recognition accuracy (pIntact | Intact - pIntact | Rearranged; see Behavioral results).

Experimental stimuli. The experimental stimuli consisted of 320 visually presented, semantically unrelated word pairs. The words were concrete nouns ranging from three to nine letters, selected from word association norms (Nelson et al., 2004). A total of 240 word pairs was presented at the time of study (encoding), with the remainder (80) held as "novel" pairs presented at test (retrieval). Of the 240 word pairs seen at study, 160 were presented at test identically to how they were shown at study (intact pairs), and 80 were "rearranged," meaning that the individual words had appeared previously but were presented at test paired with a different word. These were divided into two lists (120 pairs presented at encoding, 160 presented at test). Participants completed a practice study list consisting of a separate group of 45 word pairs shown at study, and the test list for this practice list included 60 word pairs $(30$ intact, 15 rearranged, 15 novel).

Procedure. Participants were provided instructions for both the study (encoding) task and the test period (retrieval) before undertaking the experiment proper. Upon completion of the practice session, the study and test portions of the experiment were administered. All participants completed at least one study/test cycle following practice (minimum of 
A

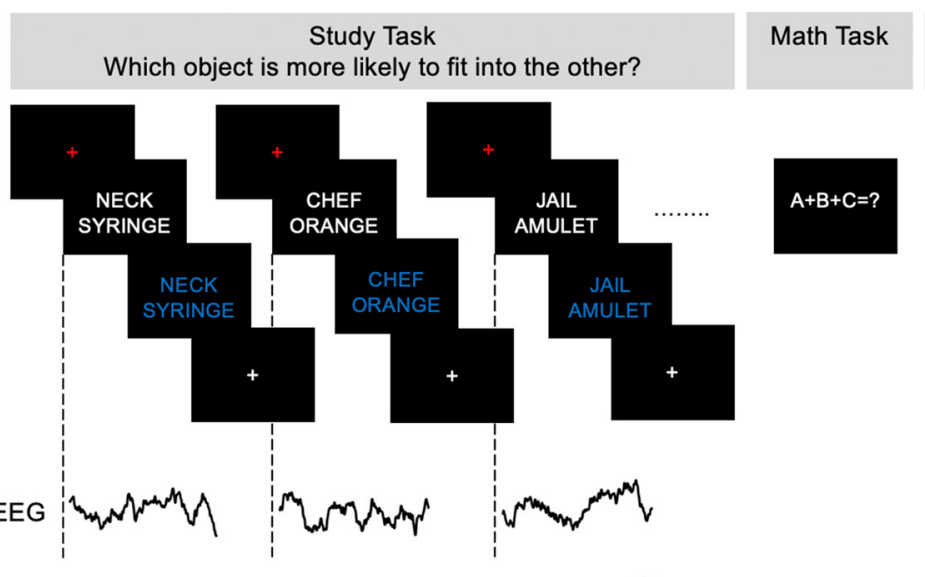

B

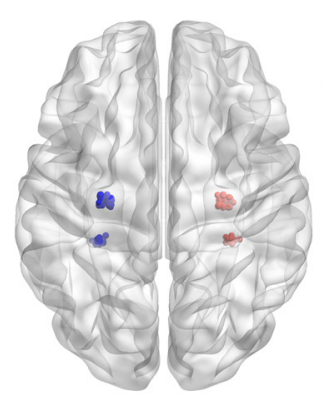

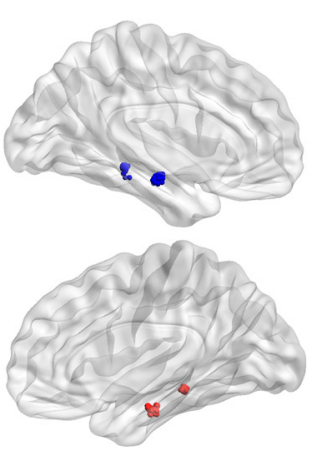

C

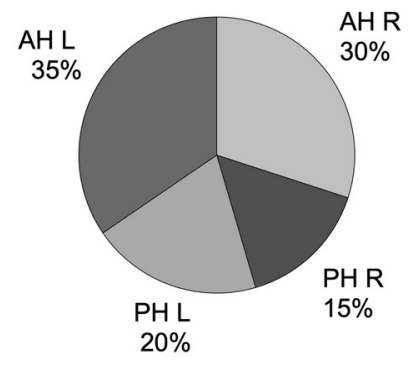

Test Task

Same, rearranged, or new?

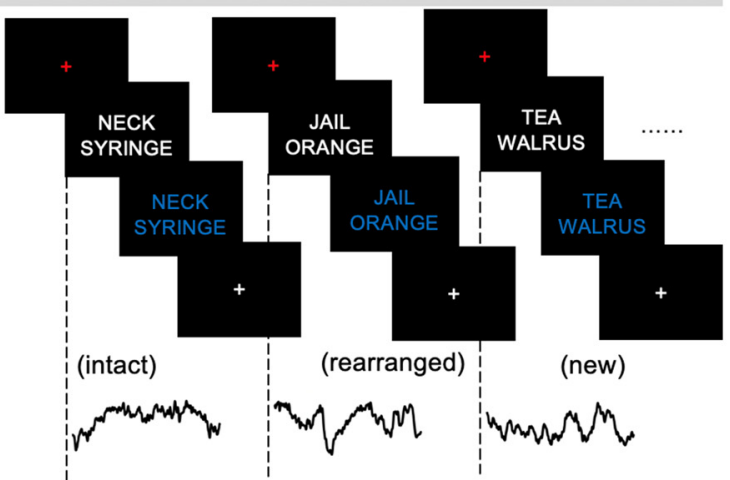

D

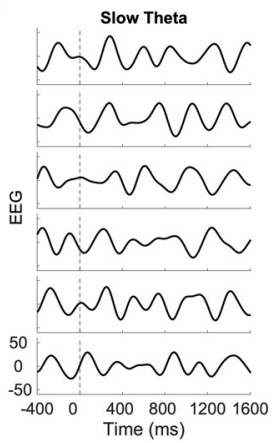

E

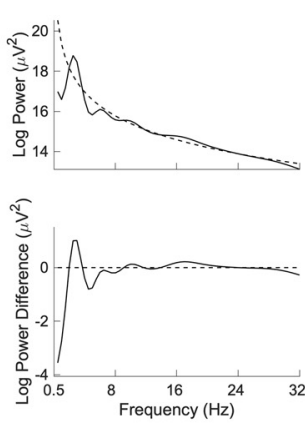

Figure 1. A, A schematic of the associative recognition paradigm showing study, distractor, and test procedures. B, Axial and sagittal view of electrode locations for anterior hippocampus and posterior hippocampus across all subjects using normalized electrode coordinates. C, Relative proportions of electrodes in the anterior hippocampus and posterior hippocampus in both hemispheres. D, Examples of single trials of slow theta EEG signal for associative hits for an anterior hippocampal recording. $\boldsymbol{E}$, A power spectrum of an associative hit trial for one of the subjects is shown in the top panel. Power spectra, after subtracting robust-fit line, are shown in the bottom panel indicating a peak in the slow theta range. AH, Anterior hippocampus; PH, posterior hippocampus; L, left; R, right.

120 word pairs at study, 160 at test). Figure $1 A$ shows a schematic overview of the study and test procedures. For the study portion, participants were required to indicate with a button press which of the two objects denoted by the words was more likely to fit into the other object. After completing the study task, participants engaged in a math distractor task for $30 \mathrm{~s}$. The task was in the form of $\mathrm{A}+\mathrm{B}+\mathrm{C}=$ ? ?, in which $\mathrm{A}, \mathrm{B}$, and $\mathrm{C}$ are single digits. Then, followed by a $5 \mathrm{~min}$ break, participants undertook the associative recognition test. For the memory test, participants were required to press one of the three keys to indicate whether a test pair was intact, rearranged, or new. "Intact" responses indicated that participants recognized both words and had a specific memory of the two words being presented together at study; rearranged responses indicated that participants recognized both words from the study but had no memory of the words having previously been presented together. If participants could not recognize one or both of the words in a test pair, they made a "new" response. The following terms are used throughout the article to describe behavioral responses: "associative hits" to denote correctly endorsed intact items; and "associative misses" to denote intact items incorrectly identified as rearranged. Study and test instructions emphasized the need for both accuracy and speed. During both study and test, participants were seated comfortably in their beds. Stimuli were presented on a laptop computer that was placed on a height-adjustable overbed bedside table $\sim 1 \mathrm{~m}$ in front of the participant.

Study and test pairs were preceded by a red fixation cross for $0.5 \mathrm{~s}$. The pairs were presented in a white uppercase Helvetica 30 font against a black background for $2 \mathrm{~s}$. Then, the study pair turned from white to blue for $1 \mathrm{~s}$ to prompt a participant's response. This same timing was used at test. Study and test pairs were followed by a white fixation cross for $1 \mathrm{~s}$ (jitter, $200 \mathrm{~ms}$ ). Reaction times (RTs) were measured from the onset of the word pairs on the laptop computer screen, but participants were allowed to respond when the font color changed from white to blue.
EEG acquisition and preprocessing. Participants all had intracranial electrodes implanted in the anterior and posterior hippocampus (17 subjects with anterior electrodes, 16 subjects with posterior electrodes, and 15 subjects contributing both left and right hemisphere hippocampal electrodes), localized by independent expert neuroradiology review, with the uncal notch set as the demarcation point between anterior and posterior regions. EEG signals from hippocampal electrodes (1-12 electrodes per hippocampus including both anterior and posterior contacts; mean $=6, S D=3$ ) were sampled at $1 \mathrm{kHz}$ on a Nihon Kohden 2100 clinical system. EEG signals were processed offline using MATLAB software (MathWorks). EEG signals were rereferenced to an average of all available electrodes (across participants, 116-260 electrodes; mean $=189$, $\mathrm{SD}=38$ ) after excluding noisy channels or those with frequent interictal activity based on the report of an epileptologist. Study pairs that were correctly endorsed as intact (subsequent associative hits) and pairs that were later incorrectly identified as rearranged (subsequent associative misses) on the subsequent associative recognition test were used in the analyses of the encoding data. Data recorded at the time of test (memory retrieval) were analyzed separately. For both the study and test phases, continuous EEG signals were segmented into $1600 \mathrm{~ms}$ epochs time locked to the onset of the stimulus. The EEG signals were notch filtered at $60 \mathrm{~Hz}$ ( $4 \mathrm{~Hz}$ bandwidth) and its harmonics. Kurtosis (ratio of fourth central moment of the data and fourth power of its SD) for each EEG epoch $(0-1600 \mathrm{~ms})$ was calculated. The EEG epochs that had a kurtosis value $>4$ were considered to contain artifacts and were excluded (Lin et al., 2017). To further eliminate artifacts, a median-based artifact detection algorithm was used (Kook et al., 2008). With this approach, the median signal of the remaining EEG epochs was calculated, and a Euclidean distance from each epoch to the median signal was then computed. The EEG epochs that exceeded the 95th percentile of the Euclidean distances were considered to be artifacts and were excluded. And the EEG epochs were resampled to $500 \mathrm{~Hz}$, and power calculated 
for the entire epoch (0-1600 ms) using Morlet wavelets at 54 logarithmic frequencies ranging from 2 to $200 \mathrm{~Hz}$ (Lin et al., 2017).

Oscillatory power analysis. To examine subsequent memory effects, power for artifact-free subsequent associative hits and subsequent associative misses were averaged across trials for every electrode in the anterior hippocampus and posterior hippocampus separately. This comparison was performed using a linear mixed-effects (subject as random effect) model across all electrodes within each hippocampus at each time point and frequency (Seltman, 2009). Events were randomly shuffled 250 times at electrode level to reduce the impact of trial imbalance, following published descriptions (Ludbrook, 1994; Files et al., 2016). False discovery rate $(\mathrm{FDR} ; \mathrm{Q}=0.05)$ was used to control the probability of type I error. Significant results in time frequency space indicate a power difference with an FDR-corrected $p$ value $<0.05$ for a minimum of one continuous cycle of an oscillation.

To further characterize slow and fast theta oscillations, power was averaged across trials for every electrode for subsequent associative hits and misses, and was normalized using $1 \mathrm{~s}$ from the prestimulus baseline period at each frequency $(2-9 \mathrm{~Hz})$. Normalized power was combined for slow theta $(2-5 \mathrm{~Hz})$ and fast theta $(5-9 \mathrm{~Hz})$ for each event for all electrodes and subjects within each hippocampus. Normalized power for subsequent associative hits versus misses at every time point ( $2 \mathrm{~ms}$ separation) was compared using a linear mixed model (with subject as a random effect) with an associated shuffle procedure (250 shuffles) at electrode level. Time points with a significant difference in normalized power after FDR $(Q=0.05)$ correction were identified.

The above analysis approach was repeated for associative hits versus associative misses in the data from the test phase (item retrieval). The FDR $(Q=0.05)$ correction was used to identify time points with a significant difference in normalized power. A pairwise analysis (matching anterior/posterior recordings within a subject) was performed using a linear mixed model (with subject as a random effect) to contrast anterior and posterior slow theta and fast theta power at retrieval for successful recollection.

Phase reset. We examined the phenomenon of phase reset for slow theta and fast theta. This analysis was performed at the electrode level incorporating subject as a random effect (Rizzuto et al., 2003, 2006; Fell et al., 2004). EEG signals were filtered using Butterworth bandpass filters of 2-5 and 5-9 Hz. Phase values were then calculated during the study phase with the Hilbert transform at every time point ( $2 \mathrm{~ms}$ separation) for subsequent associative hits and misses. A Rayleigh test was used to identify nonuniformity in the phase angle distribution at each time step across trials for each electrode (Berens, 2009). The $p$ values from this test were converted to $z$-scores using a normal inverse transformation. This process was repeated for all electrodes in the anterior and posterior hippocampus. To test whether the phase reset values ( $z$-scores) for subsequent associative hits were significantly higher than those for subsequent associative misses, a linear mixed-effects (with subject as a random effect) model was used with a $5 \%$ significance level. Time points with a significant difference in phase reset after $\operatorname{FDR}(Q=0.05)$ correction were identified. This procedure was also performed for associative hits and misses during the test phase. To test whether the slow theta phase reset that was observed for associative hits in the study and test phases tended to occur at similar phases, the mean phase difference between study and test was compared across electrodes using the Rayleigh test. The comparison was conducted separately for the anterior hippocampus and posterior hippocampus.

Reinstatement of encoding-related oscillations. Cosine similarity was used as a metric to estimate hippocampal reinstatement during successful recollection (Yaffe et al., 2014). Cosine similarity is the cosine angle between two vectors. Mathematically, it is the ratio of the dot product of two vectors and the product of Euclidean magnitudes of two vectors. It has been shown that cosine similarity and correlation methods outperform Euclidean distance for quantitating similarity between high-dimensional space vectors (Strehl et al., 2000; Huang, 2008). Pearson's correlation is similar to cosine similarity applied to the mean-centered data (Van Dongen and Enright, 2012). We conducted this analysis using a linear mixed-effects (with subject as a random effect) model. We hypothesized that cosine similarity values for successful recollection would be higher than those for failed recollection in both the anterior and posterior hippocampus. Artifact-free trials that were common to both the study and test were identified, and the power was normalized at each frequency (54 logarithmic frequencies ranging from 2 to $200 \mathrm{~Hz}$ ). For each encoding-retrieval trial, cosine similarity was calculated across the entire frequency spectrum $(2-200 \mathrm{~Hz})$, and broken down into slow theta $(2-5 \mathrm{~Hz})$ and fast theta $(5-9 \mathrm{~Hz})$ in a $500 \mathrm{~ms}$ time window with a $90 \%$ overlap (sliding window width $=50 \mathrm{~ms}$ ) for every $500 \mathrm{~ms}$ in study with all $500 \mathrm{~ms}$ time windows at the time of test following published methods (Yaffe et al., 2014). The average cosine similarity across trials was calculated for every electrode in the anterior hippocampus and posterior hippocampus. This results in a plot of reinstatement values comparing all study (encoding) windows to all test (retrieval) windows (a reinstatement map). This was done separately at each electrode for successful recollection (associative hits) and separately for unsuccessful recollection (associative misses). The Fisher $z$-transformation was applied to the cosine similarity values. The $z$-scores were compared between successful versus unsuccessful recollection across electrodes using a linear mixed-effects model at each time window. Significant differences in reinstatement magnitude were those for which the $p$ value was $<0.05$ following FDR correction across time windows. This procedure was also performed for surrogate reinstatement measure by calculating cosine similarity values for each subsequent associative hit trial with all other associative hits (Staresina et al., 2016) to compare cosine similarity values between associative hits versus nonmatching trials. This was done in an effort to test whether reinstatement is not only greater during successful associative memory formation, but also greater than that observed for nonmatching items following this previous effort.

\section{Results}

Nineteen subjects implanted with intracranial electrodes met our minimum performance threshold while completing the AR task (see Behavioral results). Further demographic details are provided in Table 1, including subjects' sex, age, duration of epilepsy (in years), hemisphere of onset, number of electrodes in hippocampus, and seizure onset location. A visual representation of electrode locations across subjects using the BrainNet Viewer (Xia et al., 2013) is shown in Figure $1 B$, and the proportion of electrodes is shown in Figure 1C. Examples of single trials of a slow theta signal for associative hits for anterior hippocampal recording are shown in Figure $1 D$. A power spectrum of an associative hit trail relative to the $1 / \mathrm{f}$ baseline (Watrous et al., 2018) and a plot showing the effect of removing the $1 / \mathrm{f}$ best fit line from a power spectrum exhibiting a peak in slow theta are shown in Figure $1 E$.

\section{Behavioral results}

Associative recognition performance was estimated by the metric $p \mathrm{R}$, the difference between the probability of an intact response to an intact test item and the probability of an intact response to a rearranged item (de Chastelaine et al., 2016a,b). Participants with a $p \mathrm{R}>0$ (probability of chance for this metric; de Chastelaine et al., 2016b) were included in the analyses presented below based on previously published methods. The mean (SD) accuracy across all item classes and RT on the study task were $0.54(0.22)$ and 2510 (85) ms, respectively. The mean (SD) proportion of intact trials correctly endorsed as intact (associative hits) was 0.59 (0.17), and a mean of 0.20 (0.09) of trials were classified as associative misses. A summary of behavioral performance on the associative recognition memory test is shown in Table 2. The mean (SD) $p \mathrm{R}$ was 0.22 (0.17). The mean RT (SD) for associative hits was 2606 (121) ms, and for associative misses, it was 2701 (184) ms. A pairwise $t$ test revealed that the RT for associative misses was significantly greater than that for associative hits $\left(t_{(18)}=3.47, p=0.0014\right)$, which was consistent with our 
Table 2. Mean (SD) proportions of intact, rearranged, and new pairs given intact, rearranged, and new responses at test

\begin{tabular}{llll}
\hline & \multicolumn{3}{l}{ Participant's response } \\
\cline { 2 - 4 } Item type at presentation & Intact & $\begin{array}{l}\text { Rearranged } \\
{[\text { [Mean (SD)] }}\end{array}$ & $\begin{array}{l}\text { Novel } \\
{[\text { SDean }(S D)]}\end{array}$ \\
\hline Intact pairs & $\mathbf{0 . 5 9 ( \mathbf { 0 . 1 7 } )}$ & $0.20(0.09)$ & $0.14(0.09)$ \\
Rearranged pairs & $0.37(0.16)$ & $\mathbf{0 . 3 4}(\mathbf{0 . 1 8})$ & $0.22(0.16)$ \\
New pairs & $0.18(0.12)$ & $0.26(0.13)$ & $\mathbf{0 . 4 8}(\mathbf{0 . 1 8})$ \\
\hline
\end{tabular}

Correctly endorsed items are highlighted in bold. Mean (SD) accuracy $=0.50$ (0.12).

expectations. After artifact rejection, the mean number of trials (range) for subsequent associates was 75 (16-125); for subsequent associative misses, it was 24 (8-52); for associative hits, it was 74 (12-125); and for associative misses, it was 23 (8-51).

\section{Theta power distinguishes successfully versus unsuccessfully} recollected items at both encoding and retrieval

Our principal hypothesis was that we would observe a difference in oscillatory power in the theta frequency range distinguishing associative hits (recollected items) from associative misses. For our initial analysis, we examined differences in power in timefrequency space. We used a linear mixed model (Seltman, 2009) with an associated shuffle procedure with FDR correction to compare oscillatory power between successfully versus unsuccessfully recollected items at each time-frequency pixel separately for the study and test phases. Significant time-frequency pixels across the $2-9 \mathrm{~Hz}$ theta range are shown in Figures $2 \mathrm{~A}$ (encoding) and $3 A$ (retrieval). During encoding, the power differences were mixed for fast theta oscillations, as we observed greater power for subsequent associative hits than for subsequent associative misses in both the anterior and posterior hippocampus centered at $\sim 600 \mathrm{~ms}$, although the opposite pattern occurred later in the time series at $1200 \mathrm{~ms}$. In the $2-5 \mathrm{~Hz}$ slow theta range, significant effects were limited to the anterior hippocampus, with greater power for subsequently recollected items.

To more precisely characterize differences in theta power during study, we contrasted normalized power extracted separately for the $2-5$ and $5-9 \mathrm{~Hz}$ frequency bands at each time point between subsequent associative hits versus subsequent associative misses. Time points at which significant differences were detected are shown in Figure $2 B$ (FDR corrected, $p<0.05$, linear mixed-effects model with a shuffle procedure). Results using this method were consistent with the results for the full time-frequency maps described above. We observed significantly greater slow theta power in the anterior hippocampus for subsequent associative hits from $\sim 550$ to $700 \mathrm{~ms}$, driven by a relatively greater normalized power decrease for subsequent misses compared with subsequent hits. While this pattern was similar between the anterior and posterior hippocampus, no time points survived FDR correction for posterior recording locations. Fast theta oscillatory power exhibited a more complex pattern, as it is significantly greater for subsequent hits from $\sim 570$ to $710 \mathrm{~ms}$ in the posterior hippocampus but relatively lower later in time following item presentation from $\sim 1250$ to $1400 \mathrm{~ms}$ in both the anterior and posterior hippocampus.

During retrieval, oscillatory differences between associative hits and misses (and between frequency bands) were easier to interpret. Figure $3 B$ shows that associative hits elicited power increases in the slow theta band that were centered at $\sim 1000 \mathrm{~ms}$ post-stimulus onset (FDR corrected $p<0.05$, linear mixedeffects model with a shuffle procedure) in the anterior hippocampus, consistent with results in the time-frequency maps illustrated in Figure $3 \mathrm{~A}$. The same pattern occurred in the posterior hippocampus, with significantly greater power centered at the same time point for associative hits in the $2-5 \mathrm{~Hz}$ band, while normalized power following the presentation of associative misses remained relatively flat across time. Dynamics for the fast theta frequency range exhibited patterns in both directions. We observed greater power for associative misses centered at $500 \mathrm{~ms}$ after item presentation in the both anterior and posterior hippocampus, but power increases that were greater for associative hits at $200 \mathrm{~ms}$ in the anterior hippocampus. To piece these findings together, we found that an increase in slow theta power uniquely distinguished associative hits from misses, whereas we observed a relatively commensurate increase in fast theta power for both classes of items (albeit with different temporal dynamics). To visualize this pattern, we directly contrast slow theta versus fast theta power for associative misses (Fig. 4), showing significantly greater fast theta relative to slow theta power following item presentation for the anterior hippocampus $(p<0.001$, linear mixedeffects model) and posterior hippocampus $(p<0.05$, linear mixed-effects model).

We also replicated our analyses at retrieval using a bipolar referencing scheme by referencing hippocampal electrodes to adjacent white matter recording locations (in the temporal lobe outside the hippocampus, localized to white matter by expert neuroradiology review). This analysis included 15 of 19 subjects who had electrodes in white matter [in the remaining 4 subjects, these contacts were not included in the recording montage because of the limited number of available amplifier input channels (256) in the clinical system]. The plot in Figure 5 indicates that power time courses are minimally different compared with our initial analysis shown in Figure $3 B$.

\section{Anterior-posterior differences at retrieval}

We compared slow theta and fast theta power for associative hits directly between the anterior and posterior hippocampus in a pairwise fashion, including only the 14 subjects who contributed simultaneous anterior and posterior recordings. Figure 6 shows the resulting contrast. Although the overall patterns are quite similar, posterior hippocampal slow theta power is significantly greater at $650-1000 \mathrm{~ms}$ (FDR corrected $p<0.05)$ after item presentation, when the maximum memory-related differences occur for this oscillation. Fast theta anterior/posterior power differences reflect different timing for when fast theta power reaches a maximum value (earlier in the posterior hippocampus, later in the anterior hippocampus).

\section{Phase reset supports associative memory formation}

We further characterized the oscillatory power effects identified in our initial analyses by examining the phenomenon of phase reset, seeking to understand whether this measure demonstrates similar or distinct patterns compared with differences in oscillatory power during encoding and retrieval. The plots in Figure 7 show the results of this analysis. Figure 7, $A$ and $B$, shows the average $z$-score extracted via Rayleigh test applied to the distribution of phase values at each time point, separately for fast and slow theta oscillations. In the anterior hippocampus, study pairs elicited both slow and fast theta-band phase reset (i.e., a significantly nonuniform distribution of phase values across trials) for subsequent associative hits but not for subsequent associative misses (Fig. 7A; FDR corrected $p<0.05$, linear mixed-effects model), a phenomenon that may have contributed to the power decrease observed for the latter items during encoding (Fig. 2A). During the test portion of the task (memory retrieval), 
A
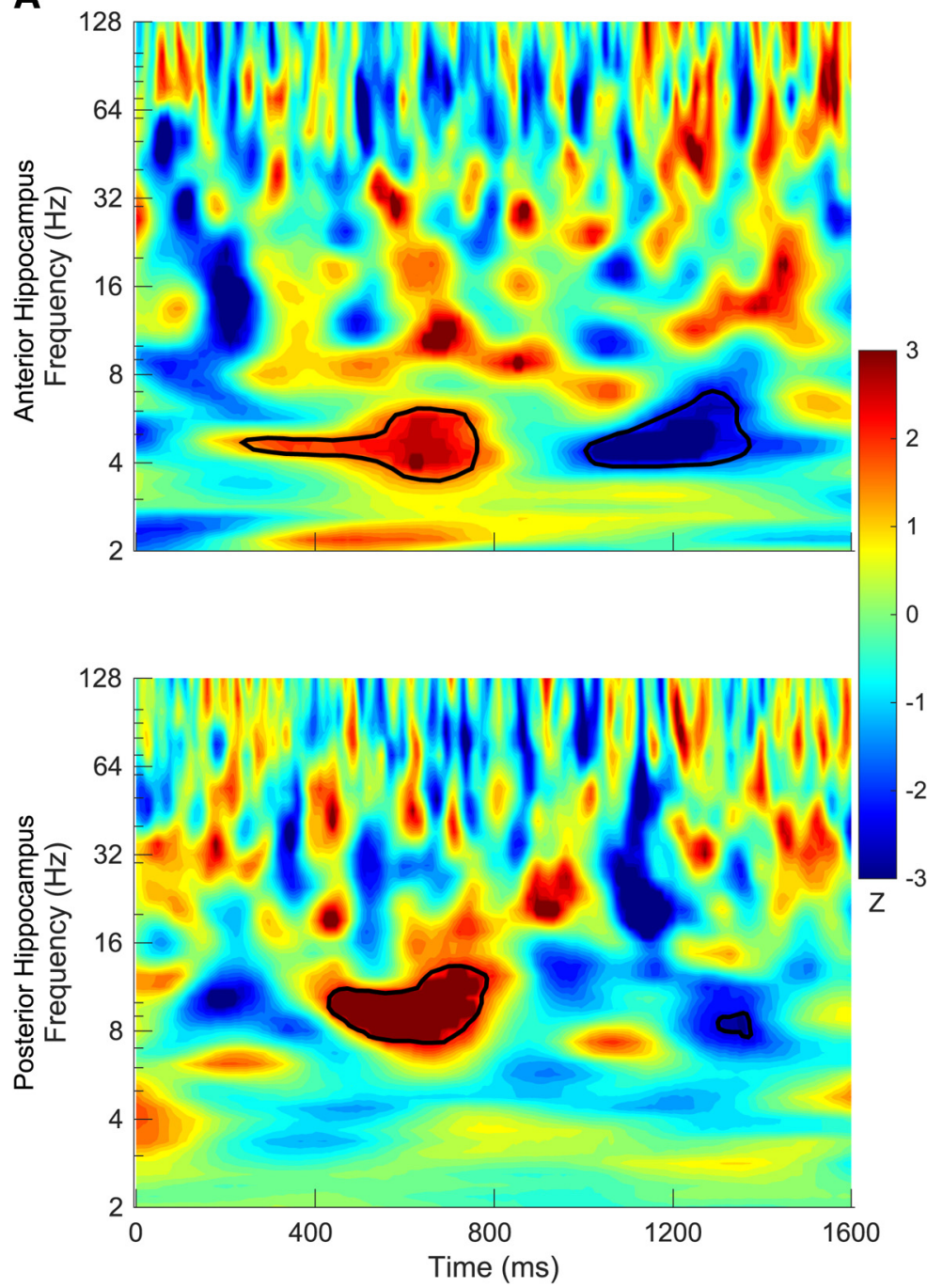

B
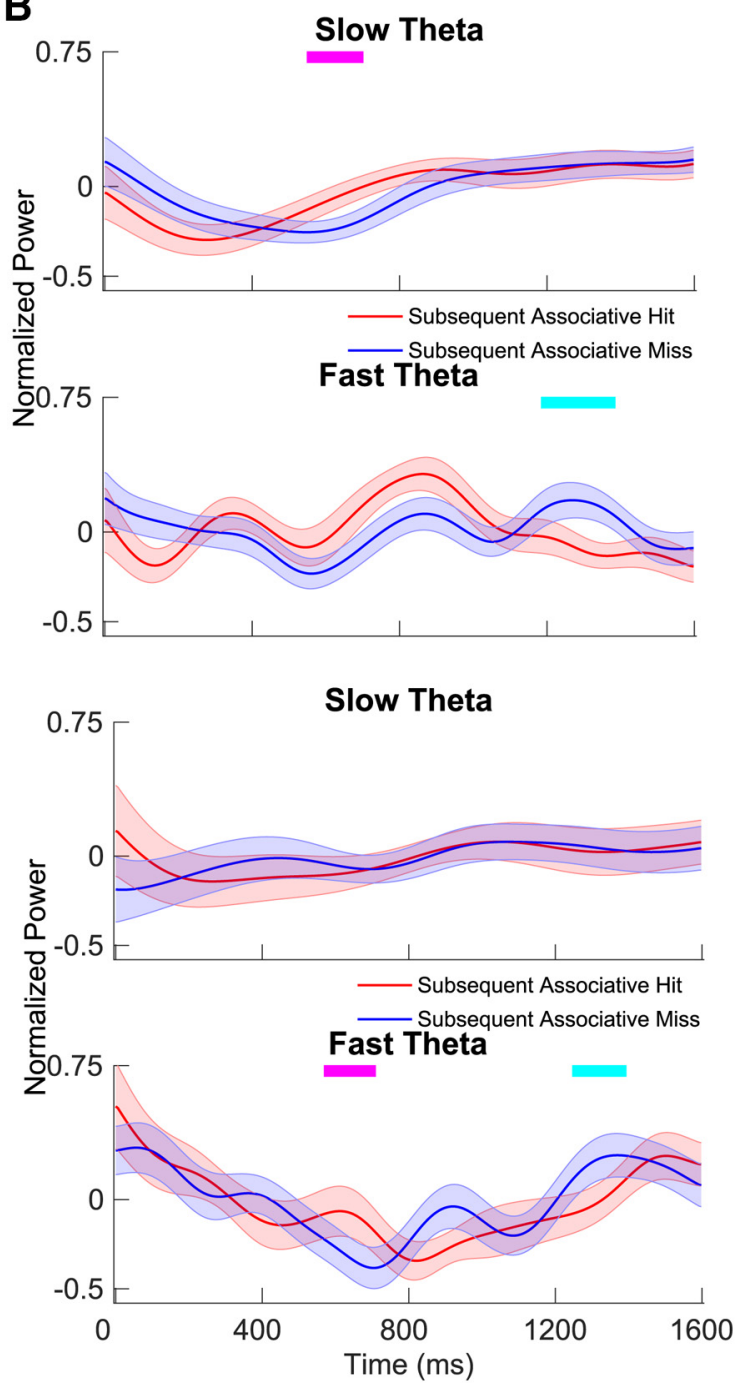

Figure 2. Time-frequency maps comparing power during encoding between intact items that went on to be classified at test as associative hits versus misses. $\boldsymbol{A}$, The $z$-scored $p$ values are plotted for anterior hippocampus (top) and posterior hippocampus (bottom). In the time-frequency map, red indicates that the power for subsequent associative hits is higher than that for subsequent associative miss, and blue indicates the opposite. Time-frequency pixels in the shaded region in theta range (2-9 Hz) survived correction (FDR corrected $p<0.05$, random-effects model with a permutation procedure across stimulus conditions). $\boldsymbol{B}$, Aggregate normalized power for slow theta and fast theta for the anterior hippocampus (top) and posterior hippocampus (bottom). Tick marks on the top in magenta indicate time points at which normalized power for subsequent associative hits is significantly higher than subsequent associative misses using a random-effects model with a permutation procedure (FDR corrected $p<0.05$ ), and tick marks in cyan indicate the opposite. Shading represents the standard error of the mean (SEM) across electrodes.

associative hits elicited significantly greater phase reset than misses (FDR corrected $p<0.05$, linear mixed-effects model) in both the anterior and posterior hippocampus for the slow theta oscillation (Fig. $7 B$ ). While phase reset was significant across subjects, we did not observe a consistent and specific phase to which oscillations were resetting for successful recollection (no significant clustering of the mean preferred phase to which reset occurred; Fig. 7). The pattern of phase reset was different in the fast theta band during memory retrieval. For fast theta oscillations, the magnitude of phase reset was less apparent during memory retrieval for associative hits, although we observed significant differences in both directions (greater for associative hits and misses at different time points).

We next examined whether the slow theta phase reset observed for associative hits during encoding and retrieval tended to occur at similar or different phases of the theta oscillation, motivated by previous findings in the human neocortex and modeling approaches (SPEAR System) that predict distinct theta phases for ensemble activity (Hasselmo and Stern, 2014). We compared the difference in the mean phase across study and test trials at the time segment of peak phase reset in a pairwise manner (calculating the difference in mean phase for each recording location). The resulting distribution (of phase differences) is shown in Figure $7 D$. For the anterior hippocampus, the phase difference between encoding and retrieval was significantly nonuniform, though it was relatively close to zero ( $m e a n=35^{\circ}$, consistent with similar phase values during both encoding and retrieval; $p<0.001$, Rayleigh test), while for the posterior hippocampus the difference was $-95^{\circ}$, which is more consistent with the predictions of the SPEAR System of an encoding/retrieval phase difference ( $p=0.02$, Rayleigh test). The distribution of the phase difference between encoding and retrieval was significantly different between the anterior hippocampus and posterior hippocampus (Watson-Williams test, $p<0.001$ ). These findings suggest that a greater reset of phase occurs during successful associative memory formation, and the angles of reset are similar between encoding and retrieval for the anterior hippocampus 
A
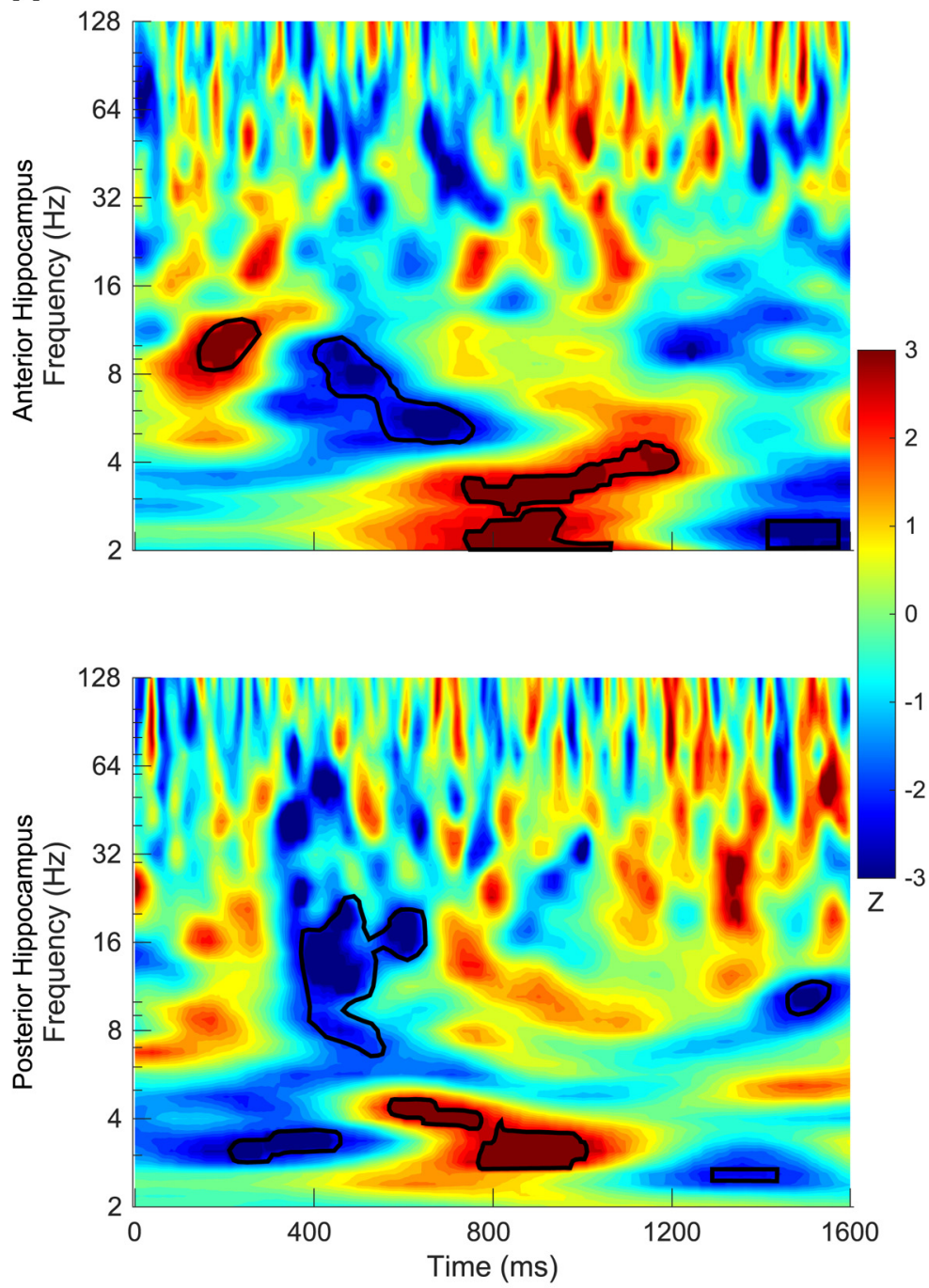

B

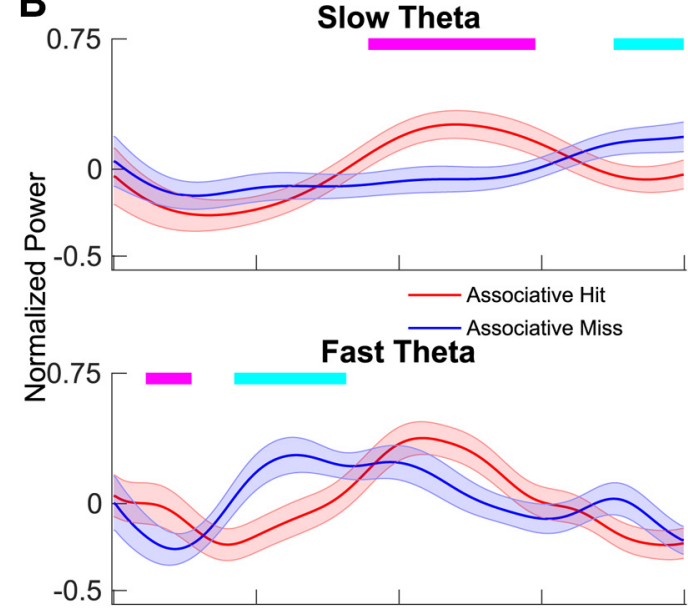

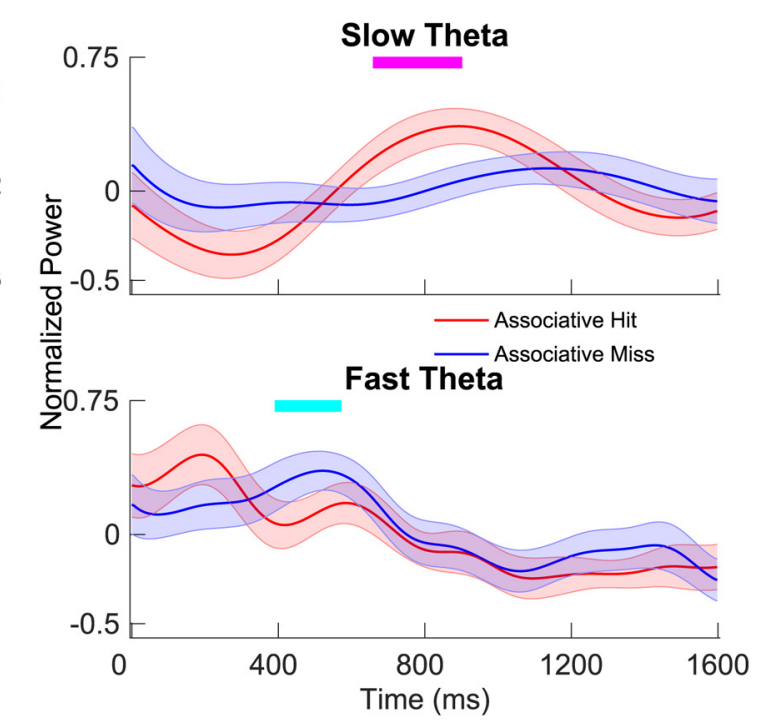

Figure 3. Time-frequency maps comparing power during retrieval between associative hits and misses. $\boldsymbol{A}$, The $z$-scored $p$ values are plotted for anterior hippocampus (top) and posterior hippocampus (bottom). In the time-frequency map, red indicates that the power for associative hits is higher than that for associative misses, and blue indicates the opposite. Time-frequency pixels in the shaded region in theta range $(2-9 \mathrm{~Hz}$ ) survived correction (FDR corrected $p<0.05$, random-effects model with a permutation procedure across stimulus conditions). $\boldsymbol{B}$, Aggregate normalized power for slow theta and fast theta for the anterior hippocampus (top) and posterior hippocampus (bottom). Tick marks on the top in magenta indicate time points at which normalized power for associative hits is significantly higher than associative misses using a random-effects model with a permutation procedure (FDR corrected $p<0.05$ ), and tick marks in cyan indicate the opposite. Shading represents the SEM across electrodes.
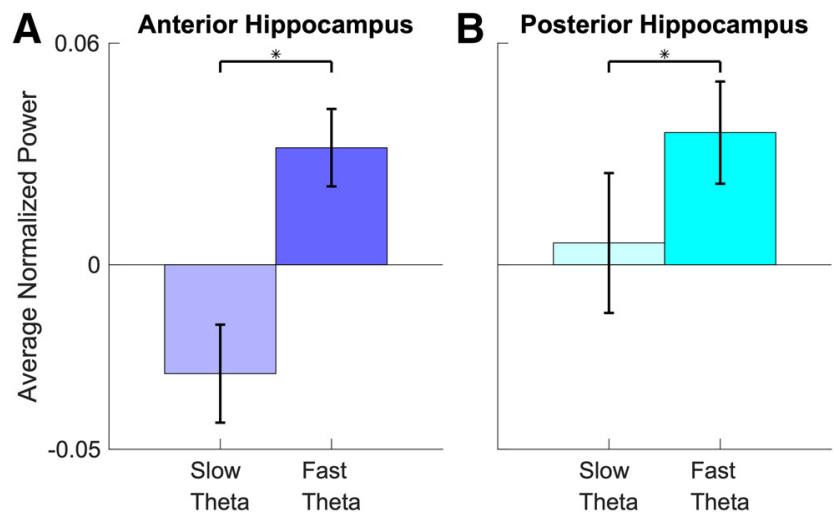

Figure 4. $\quad \boldsymbol{A}, \boldsymbol{B}$, Bar plots show the averaged normalized power for slow theta and fast theta across poststimulus interval $(0-1600 \mathrm{~ms})$ for associative misses for anterior hippocampus $(\boldsymbol{A})$ and posterior hippocampus $(\boldsymbol{B})$. The asterisk indicate the power for fast theta oscillations is significantly higher than that for slow theta oscillations $(p<0.05$, random-effects model). Error bars indicate the SEM across electrodes. (phase difference near zero), but different in the posterior hippocampus (phase difference of $-95^{\circ}$ ).

\section{Reinstatement of encoding-related oscillations is stronger for recollected items}

We measured the magnitude of pattern reinstatement of oscillatory activity using cosine similarity. Reinstatement quantifies the patterns of brain activity elicited by a given item at both encoding and retrieval. We used the cosine similarity measure based on its previous application to human intracranial data (Yaffe et al., 2014). We performed this for associative hits and misses in both anterior and posterior hippocampus (Fig. $8 A, B$, first and second columns). We compared the magnitude of reinstatement using normalized power from across the $2-200 \mathrm{~Hz}$ frequency spectrum, as well as specifically in the slow and fast theta frequency bands (Fig. 8). Time points at which reinstatement was significantly greater for recollected items are highlighted (FDR corrected $p<0.05$, linear mixed-effects model). High cosine 
similarity values along the diagonal reflect encoding-retrieval overlap at the same poststimulus latencies, while the edges in off-diagonal of the plot represent encoding and retrieval epochs that are not closely matched in time. We separately tested for memory-related effects in reinstatement magnitude for slow theta and fast theta oscillations specifically, identifying functional differences that were similar in both bands, with greater reinstatement along the diagonal for associative hits. We noted that these functional effects were stronger in the posterior hippocampus (Fig. $8 B$, fourth column). To quantify anterior-posterior differences, we compared the total number of pixels that exhibited functionally significant reinstatement (greater for associative hits; Fig. 8B, warm colors on the plot) for the two hippocampal subregions. By this metric, reinstatement was greater in the posterior hippocampus $\left(\chi_{(1,1058)}^{2}=28\right.$, $p<0.0001)$ for the entire frequency spectrum $(2-200 \mathrm{~Hz})$. To further validate the above findings, we used a surrogate reinstatement measure by calculating cosine similarity values for each subsequent associative hit trial with all other associative hits (Fig. 8A,B, third column). The cosine similarity values for successfully recollected trials were significantly greater than the surrogate trials (Fig. $8 A, B$, fifth column) at most pixels overlapping with the functional comparison. Overall, these findings were consistent with previous reports in human data (Staresina et al., 2016), although we examine the possible ramifications of longitudinal differences in the Discussion section.

\section{Discussion}

We used an associative recognition memory task in humans coupled with intracranial recordings, allowing us to directly test whether theta oscillatory activity distinguishes successful versus unsuccessful associative encoding and retrieval. The nature of the task ensures a process pure measurement of brain activity linked with associative information, which seems to elicit strong hippocampal activation in noninvasive data (de Chastelaine et al., 2016a,b). We sought to examine a central prediction of mnemonic models (Buzsáki, 2002; Poppenk et al., 2013) that theta oscillations should preferentially support associative memory at the time of study (encoding) and test (retrieval). Our key findings were as follows: (1) that power in the $2-5 \mathrm{~Hz}$ range reflected processes supporting associative rather than nonassociative memory especially during item retrieval, with weaker effects during encoding; (2) that this power difference occurred following phase reset of theta oscillations elicited by the onset of the study and test items; (3) that the strength of reinstatement of oscillatory patterns distinguished associative hits from misses, especially in the posterior hippocampus; and (4) that slow theta power during recollection was significantly greater in the posterior hippocampus, though the patterns were similar between the two regions. These observations directly link theta-range activity to associative memory encoding and retrieval, and establish a behaviorally relevant contrast between $2-5 \mathrm{~Hz}$ slow theta and 5$9 \mathrm{~Hz}$ fast theta oscillations.
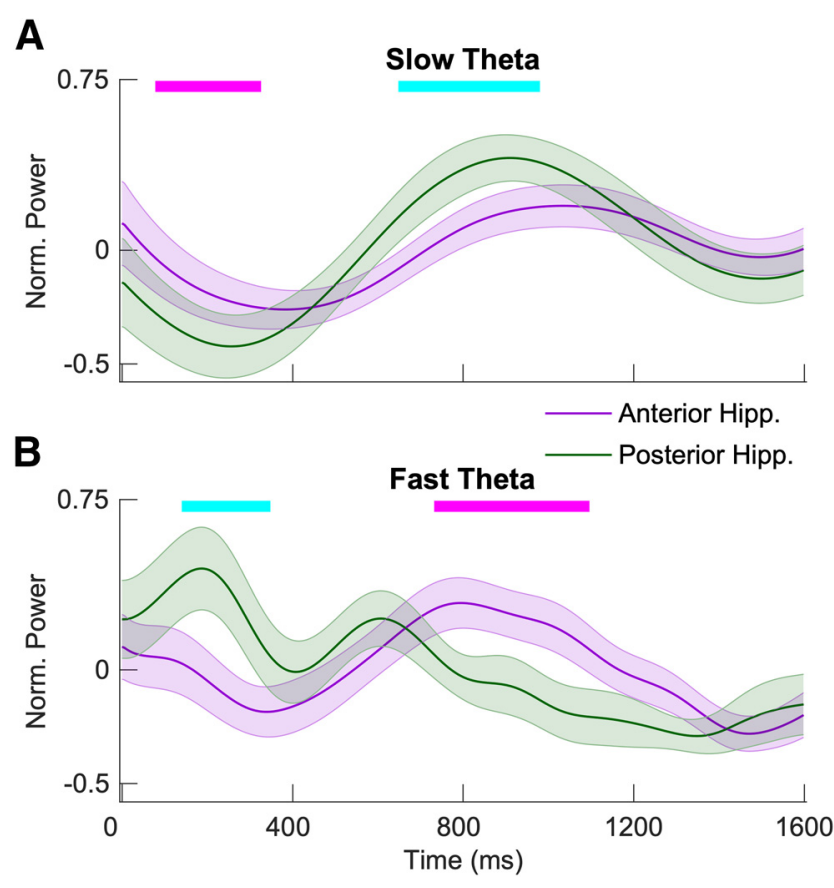

Figure 6. $\quad \boldsymbol{A}, \boldsymbol{B}$, Aggregate normalized power for anterior hippocampus and posterior hippocampus for slow theta $(\boldsymbol{A})$ and fast theta $(\boldsymbol{B})$ for successful recollection. Tick marks on the top in magenta indicate time points at which normalized power for anterior hippocampus is significantly higher than that for posterior hippocampus using a random-effects model (FDR corrected $p<0.05$ ), and tick marks in cyan indicate the opposite. Shading represents the SEM across electrodes.

Models of associative memory posit that theta oscillations in the hippocampus and neocortex provide a critical binding function to link together activity across cortical areas involved in the spatially (and temporally) distributed representation of event features (Staudigl and Hanslmayr, 2013; Backus et al., 2016). The aim of clarifying the mnemonic role of human hippocampal theta oscillations motivated us to implement the present 
A

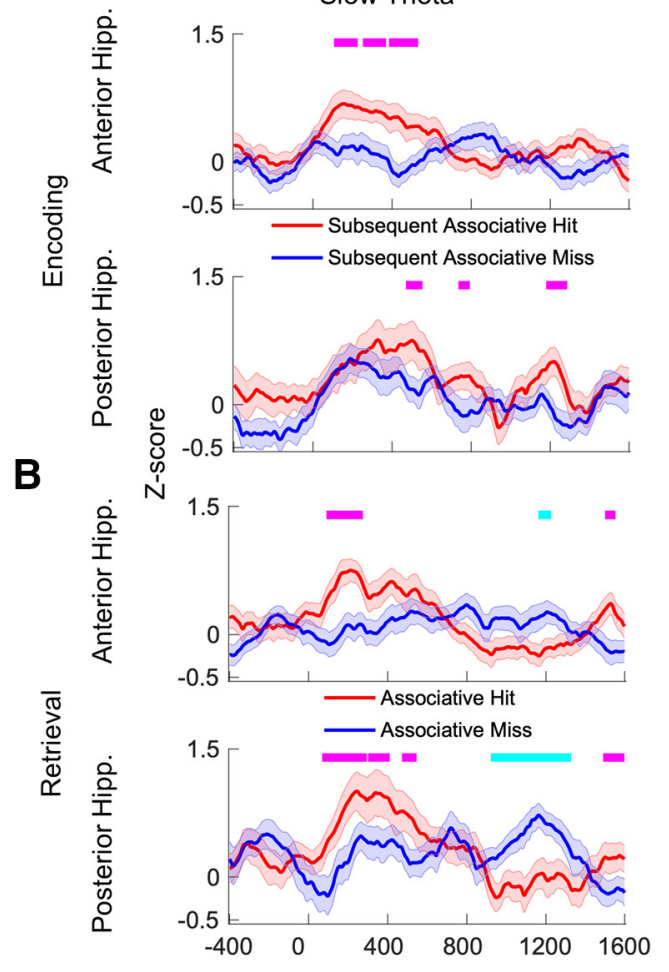

Fast Theta
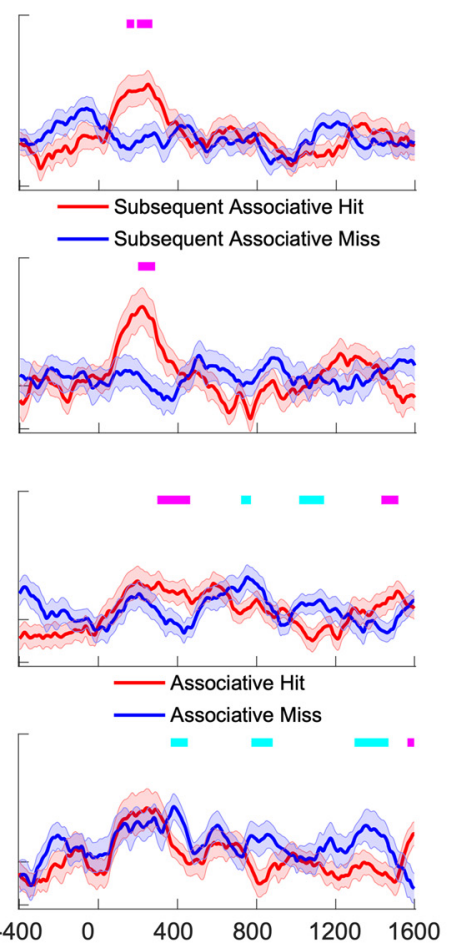

C

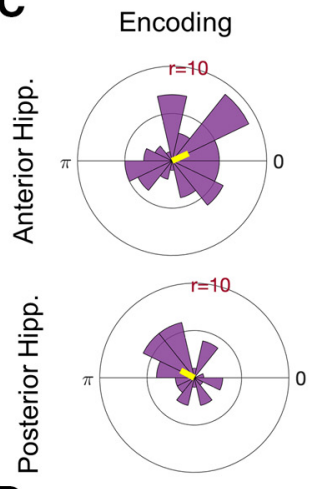

Phase difference encoding vs retrieval

Retrieval
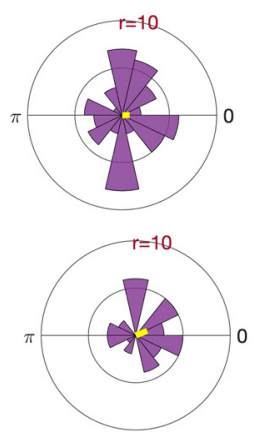

$=10$

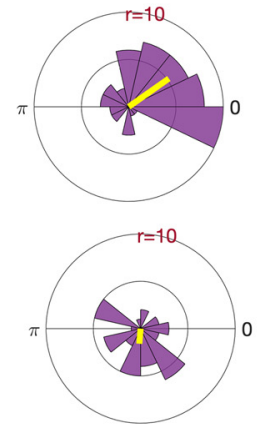

Figure 7. Phase reset during encoding and retrieval. $\boldsymbol{A}, \boldsymbol{B}$, Average $\boldsymbol{z}$-score values from Rayleigh test across all electrodes during encoding $(\boldsymbol{A})$ and retrieval $(\boldsymbol{B})$ are shown for the anterior hippocampus (top) and posterior hippocampus (bottom). Tick marks on the top in magenta indicate time points at which the $z$-score for associative hits is significantly higher than for associative misses using a random-effects analysis (FDR corrected $p<0.05$ ), and tick marks in cyan indicate the opposite. C, The polar histograms show the distribution of average phase values to which reset occurred across all electrodes for slow theta in anterior hippocampus (top) and posterior hippocampus (bottom). $\boldsymbol{D}$, Histograms of the difference in average phase values for encoding versus retrieval for slow theta oscillations. The average difference is $35^{\circ}$ for the anterior hippocampus (top) and $-95^{\circ}$ for the posterior hippocampus (bottom).

associative recognition memory paradigm coupled with direct brain recordings.

A key outstanding ambiguity regarding hippocampal theta oscillations in humans is whether there are functional differences between oscillations in the $2-5$ and $5-9 \mathrm{~Hz}$ frequency ranges. In our analysis, the most profound differences in memory-related patterns between slow and fast theta oscillations occurred during memory retrieval. As shown in Figure 3, we found that slow theta, but not fast theta, oscillations exhibit an increase in oscillatory power that is unique to associative hits. Figures 3 and 4 demonstrate that this pattern emerges because both associative hits and misses elicit increases in fast theta power, but associative hits uniquely elicit slow theta power increases. These observations are consistent with a specific association between slow theta power increases and successful associative memory formation at the time of retrieval. Our findings do not, however, support a model in which slow theta oscillations exclusively support associative memory formation at both encoding and retrieval: like slow oscillations, fast theta oscillations also exhibited a phase reset memory effect in both the anterior and posterior hippocampus during encoding. Further, the timing of fast theta oscillatory peaks relative to stimulus presentation (in the anterior hippocampus) may also distinguish associative hits from misses (Fig. 3B). Understanding different connectivity relationships for these two oscillations using the associative recognition paradigm may help to illuminate separate slow and fast theta networks that explicate regions involved in successful versus unsuccessful recollection, especially during retrieval. However, our findings help explain some of the mixed findings observed using intracranial EEG among different theta frequency ranges (Mormann et al.,
2005; Axmacher et al., 2010; Lega et al., 2012; Chaieb et al., 2015; Lin et al., 2017). We note that the use of the term "slow theta" to describe the memory-relevant oscillatory activity as opposed to "delta" oscillations is not universally adopted. We do not attempt to identify distinct sub- $2 \mathrm{~Hz}$ delta oscillations and differentiate these from slow theta oscillations, as our paradigm is not well suited to distinguishing slight differences in delta versus slow theta activity. This is related to the temporal duration of activity following item presentation, especially compared with previous analyses in spatial navigation paradigms with extended behavioral epochs that have examined this question (Bohbot et al., 2017). We also note that the time-frequency maps in Figures 2 and 3 suggest that non-theta frequency oscillations may distinguish associative from nonassociative memory. Contrasting patterns in gamma or alpha bands ranges with those observed in slow and fast theta bands remain an active area for further investigation. The study findings suggest that theta oscillatory activity in the hippocampus facilitates associative memory, but no strong conclusions can be made on recollection versus familiarity or dual-process versus single-process theories. Additional studies dedicated to comparisons of associative misses and correct rejections (correctly rejected novel trials at test) will be necessary to address this question more directly.

Our findings suggest that slow theta phase reset occurs during both encoding and retrieval, supporting its importance for associative encoding. The pattern for fast theta oscillations is more nuanced, as these oscillations exhibited phase reset during encoding but not retrieval for associative hits. Theta phase reset represents a possible mechanism for synchronizing single-unit activity in the hippocampus, facilitating long-term potentiation and formation of neuronal ensembles representing features of a 
Anterior Hippocampus

\section{Associative Hit}
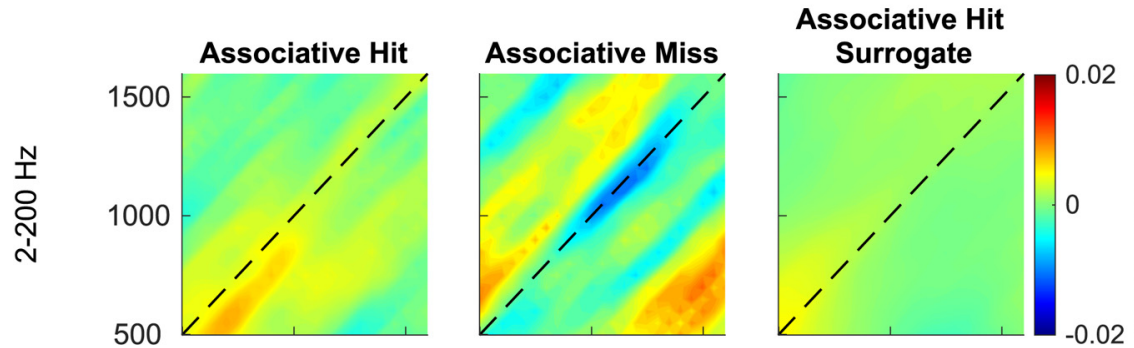

Hit vs. Miss
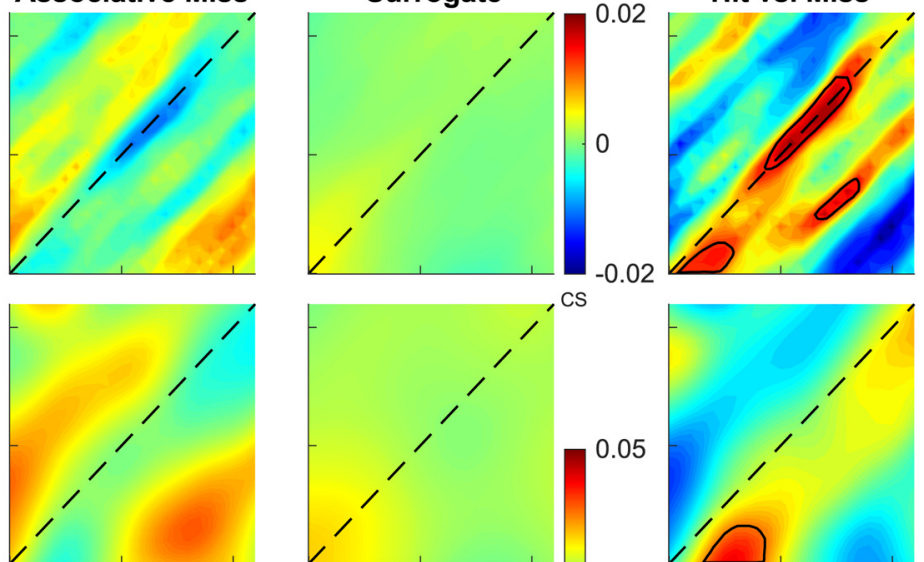

500
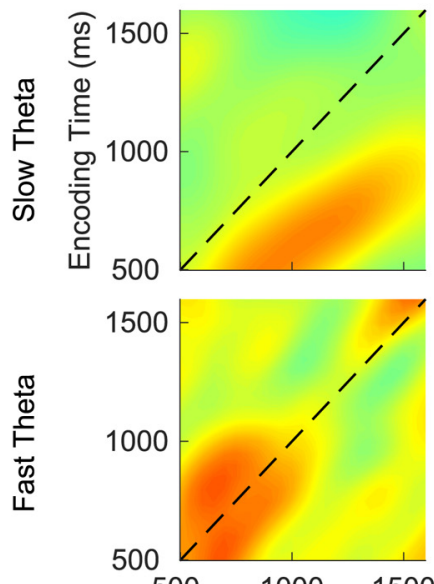

$500 \quad 1000 \quad 1500500$

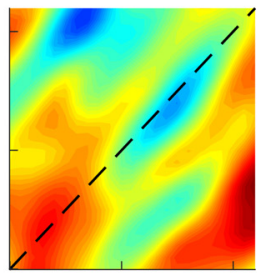

1000
1500500
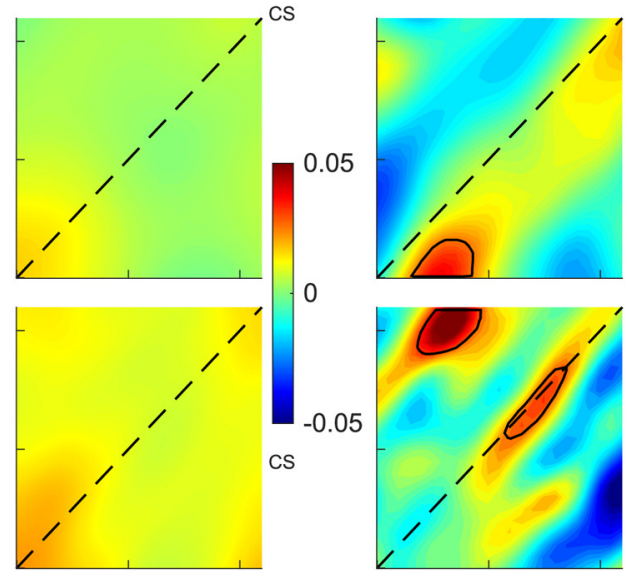

$1000 \quad 1500$

Retrieval Time (ms)

Posterior Hippocampus

B

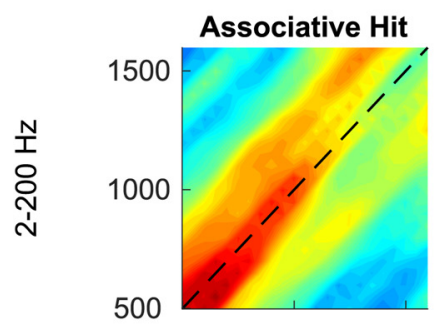

高
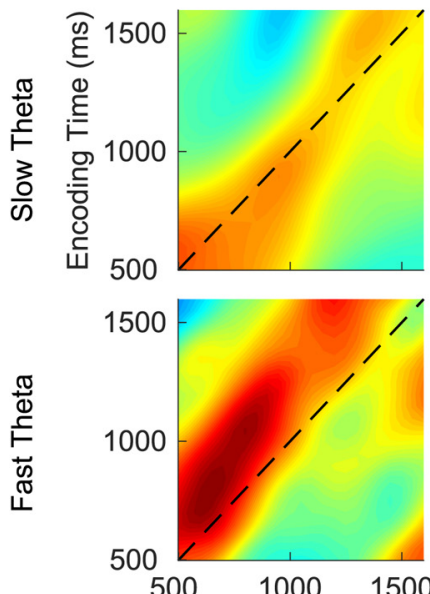
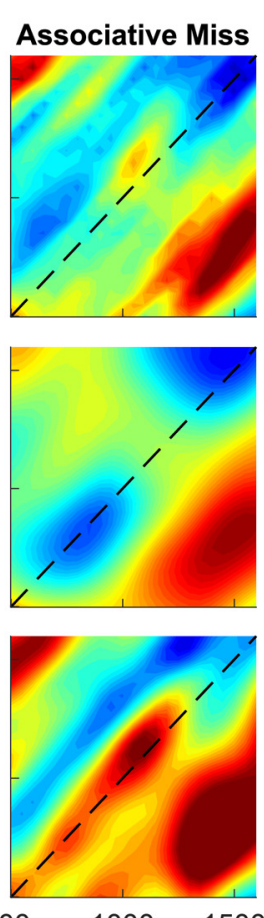

Associative Hit

\section{Surrogate}
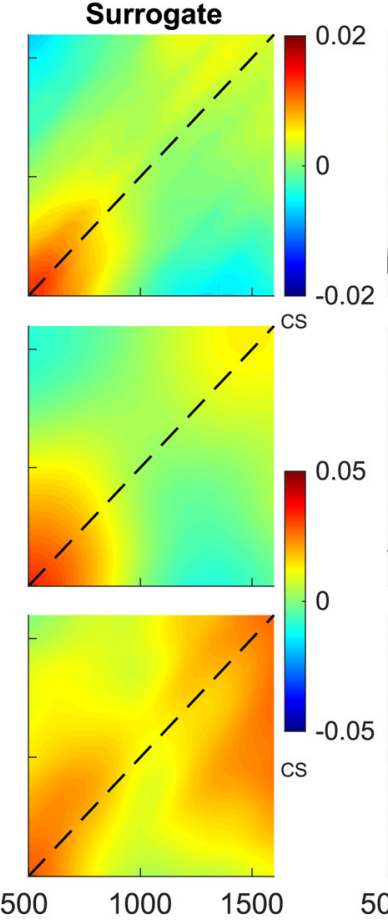

Retrieval Time (ms)

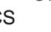

Hit vs. Miss
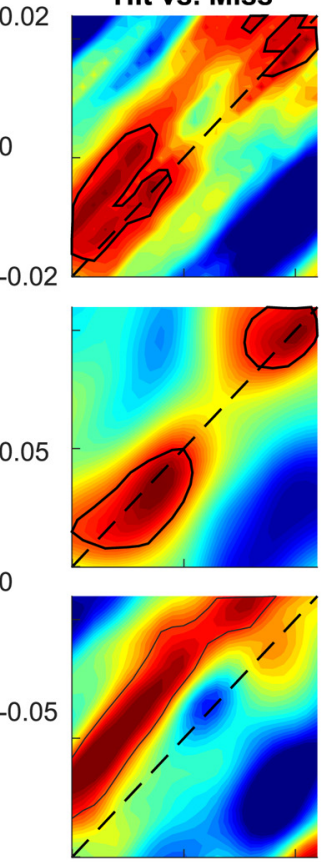

500
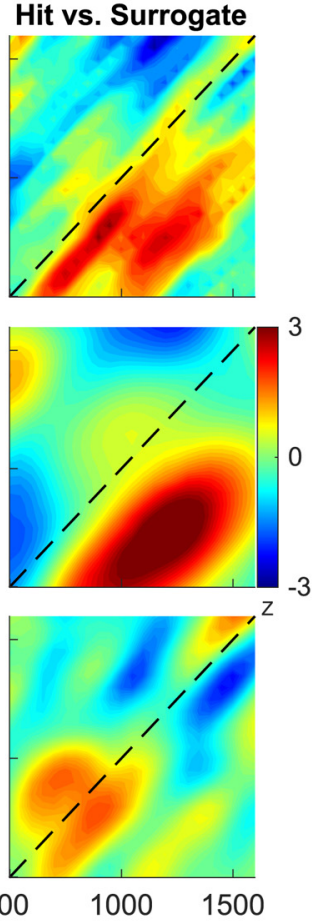
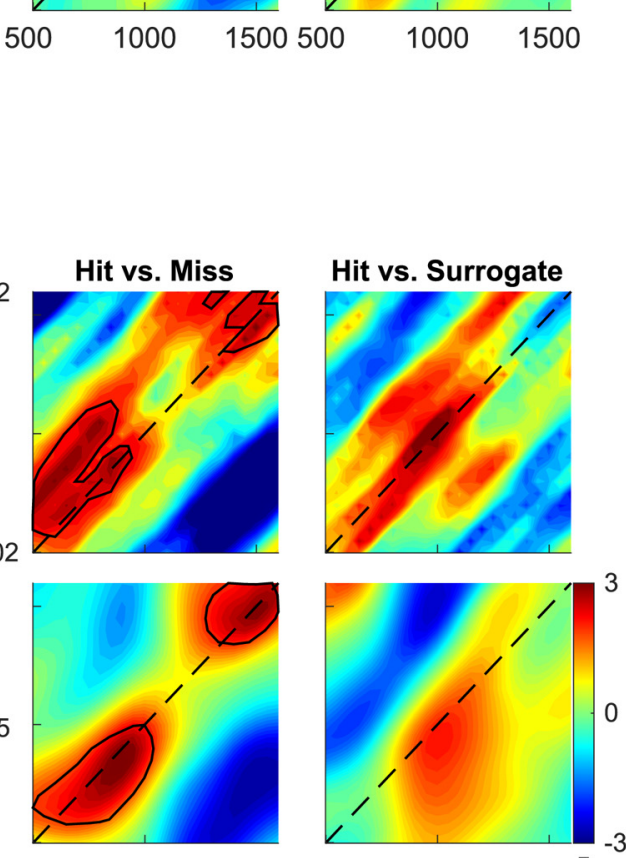

Figure 8. Reinstatement of encoding-related oscillations. $\boldsymbol{A}, \boldsymbol{B}$, Average cosine similarity across all electrodes and subjects between encoding and retrieval for associative hits, misses, surrogates of associative hits for anterior hippocampus $(\boldsymbol{A})$ and posterior hippocampus $(\boldsymbol{B})$ for all frequencies $(2-200 \mathrm{~Hz})$, slow theta, and fast theta bands are shown. The plots in the fourth and fifth column depict $z$-score from a random-effects analysis for associative hits versus misses and associative hits versus surrogates. Red indicates that reinstatement for associative hits is significantly higher than that associative misses (fourth column) and surrogates (fifth column), and blue indicates the opposite. Warm-colored pixels in the shaded region indicate survived FDR correction. 
memory event (Jutras and Buffalo, 2010). Intracranial recordings in nonhuman primates performing a recognition memory test also suggest that stimulus-induced reset of the phase of fast theta oscillations (centered at $8 \mathrm{~Hz}$ ) at the time of encoding predicts retrieval success (Killian et al., 2015). In human neocortical recordings, recognition memory items elicited phase reset across a broad frequency range that included fast theta oscillations (Rizzuto et al., 2006). The results in these experiments supported predictions of the SPEAR model articulated by Hasselmo and Stern (2014), in that there was a consistent offset between the preferred phase for phase reset observed at encoding versus retrieval. Applying a similar analytical approach to our data, we found evidence of a dissociation between anterior and posterior hippocampal phase reset. The encoding/retrieval difference in the preferred phase for a reset in the anterior hippocampus was close to 0 , but in the posterior hippocampus was $-95^{\circ}$, which is more consistent with the SPEAR model, which posits that the theta phase at which ensemble activity occurs distinguishes encoding versus retrieval. Our findings are most consistent with a model by which the retrieval of associative memories elicits the reset of an ongoing oscillation rather than including oscillations de novo during every trial, mainly because the timing of phase reset for both fast and slow theta bands does not match when we observe changes in oscillatory power (compare Figs. $3 B, 7 B$ ). Fully disambiguating these possibilities may require alternate analytical approaches, although our findings support previous publications arguing that phase reset and power effects are distinct and dissociable during memory formation (Fell et al., 2004).

Reinstatement effects were stronger for associative hits than for misses, especially in the posterior hippocampus across all frequencies. This finding supports the widely held assumption that the accuracy of associative memory retrieval depends on the rerepresentation of oscillatory features present at encoding (Rugg et al., 2008), and models of mnemonic processing by which vivid representations characteristic of episodic memories occur because of hippocampal pattern completion (Hanslmayr et al., 2016). Compared with previous iEEG studies (Staresina et al., 2016), our findings explicitly contrast reinstatement magnitudes for slow and fast theta oscillations, as well as contrasting functional reinstatement effects in the anterior and posterior hippocampus. Our findings are also consistent with a prior human iEEG study reporting that memory reinstatement was identifiable in hippocampal low-frequency oscillations $(1-8 \mathrm{~Hz})$ during a spatial navigation task (Pacheco Estefan et al., 2019) as well as noninvasive data using similar paradigms (Liang and Preston, 2017).

Our data were consistent with previous findings regarding functional dissociation along the hippocampal longitudinal axis. While the key patterns in anterior and posterior locations were similar in general terms, theta power during associative hits was significantly greater in the posterior hippocampus, consistent with previous findings observed during episodic memory encoding in a free recall paradigm (Lin et al., 2017). While our data cannot inform the discussion of models of "granularity" of information representation along the hippocampal axis (Poppenk et al., 2013), relatively stronger posterior theta power and pattern reinstatement distinguishing recollected items may support differential hippocampal contributions in the posterior medial/anterior temporal networks (Ritchey et al., 2015). Overall, however, the presence of memory-sensitive theta oscillatory activity in both the anterior and posterior hippocampus suggests that models focusing exclusively on emotional content (favoring anterior activation) or spatial memory (favoring posterior activation) remain somewhat incomplete (Poppenk et al., 2013).

\section{References}

Axmacher N, Mormann F, Fernández G, Elger CE, Fell J (2006) Memory formation by neuronal synchronization. Brain Res Rev 52:170-182.

Axmacher N, Henseler MM, Jensen O, Weinreich I, Elger CE, Fell J (2010) Cross-frequency coupling supports multi-item working memory in the human hippocampus. Proc Natl Acad Sci U S A 107:3228-3233.

Backus AR, Schoffelen JM, Szebényi S, Hanslmayr S, Doeller CF (2016) Hippocampal-prefrontal theta oscillations support memory integration. Curr Biol 26:450-457.

Berens P (2009) CircStat: a MATLAB toolbox for circular statistics. J Stat Softw 31:1-21.

Bohbot VD, Copara MS, Gotman J, Ekstrom AD (2017) Low-frequency theta oscillations in the human hippocampus during real-world and virtual navigation. Nat Commun 8:14415.

Buzsáki G (2002) Theta oscillations in the hippocampus. Neuron 33:325340.

Chaieb L, Leszczynski M, Axmacher N, Höhne M, Elger CE, Fell J (2015) Theta-gamma phase-phase coupling during working memory maintenance in the human hippocampus. Cogn Neurosci 6:149-157.

de Chastelaine M, Mattson JT, Wang TH, Donley BE, Rugg MD (2016a) The neural correlates of recollection and retrieval monitoring: relationships with age and recollection performance. Neuroimage 138:164-175.

de Chastelaine M, Mattson JT, Wang TH, Donley BE, Rugg MD (2016b) The relationships between age, associative memory performance, and the neural correlates of successful associative memory encoding. Neurobiol Aging 42:163-176.

Eichenbaum H (2017) Prefrontal-hippocampal interactions in episodic memory. Nat Rev Neurosci 18:547-558.

Ekstrom AD, Caplan JB, Ho E, Shattuck K, Fried I, Kahana MJ (2005) Human hippocampal theta activity during virtual navigation. Hippocampus 15:881-889.

Fell J, Axmacher N (2011) The role of phase synchronization in memory processes. Nat Rev Neurosci 12:105-118.

Fell J, Dietl T, Grunwald T, Kurthen M, Klaver P, Trautner P, Schaller C, Elger CE, Fernández G (2004) Neural bases of cognitive ERPs: more than phase reset. J Cogn Neurosci 16:1595-1604.

Fellner MC, Volberg G, Wimber M, Goldhacker M, Greenlee MW, Hanslmayr S (2016) Spatial mnemonic encoding: theta power decreases and medial temporal lobe BOLD increases co-occur during the usage of the method of loci. eNeuro 3:ENEURO.0184-16.2016.

Files BT, Lawhern VJ, Ries AJ, Marathe AR (2016) A permutation test for unbalanced paired comparisons of global field power. Brain Topogr 29:345-357.

Greenberg JA, Burke JF, Haque R, Kahana MJ, Zaghloul KA (2015) Decreases in theta and increases in high frequency activity underlie associative memory encoding. Neuroimage 114:257-263.

Hanslmayr S, Staresina BP, Bowman H (2016) Oscillations and episodic memory: addressing the synchronization/desynchronization conundrum. Trends Neurosci 39:16-25.

Hasselmo ME, Stern CE (2014) Theta rhythm and the encoding and retrieval of space and time. Neuroimage 85:656-666.

Huang A (2008) Similarity measures for text document clustering. Paper presented at Sixth New Zealand Computer Science Research Student Conference (NZCSRSC 2008), Christchurch, New Zealand, April.

Jacobs J (2014) Hippocampal theta oscillations are slower in humans than in rodents: implications for models of spatial navigation and memory. Philos Trans R Soc Lond B Biol Sci 369:20130304.

Jutras MJ, Buffalo EA (2010) Synchronous neural activity and memory formation. Curr Opin Neurobiol 20:150-155.

Killian NJ, Potter SM, Buffalo EA (2015) Saccade direction encoding in the primate entorhinal cortex during visual exploration. Proc Natl Acad Sci U S A 112:15743-15748.

Kook H, Gupta L, Kota S, Molfese D, Lyytinen H (2008) An offline/real-time artifact rejection strategy to improve the classification of multi-channel evoked potentials. Pattern Recognit 41:1985-1996.

Lega B, Burke J, Jacobs J, Kahana MJ (2016) Slow-theta-to-gamma phase-amplitude coupling in human hippocampus supports the formation of new episodic memories. Cereb Cortex 26:268-278.

Lega BC, Jacobs J, Kahana M (2012) Human hippocampal theta oscillations and the formation of episodic memories. Hippocampus 22:748-761.

Liang JC, Preston AR (2017) Medial temporal lobe reinstatement of contentspecific details predicts source memory. Cortex 91:67-78. 
Lin JJ, Rugg MD, Das S, Stein J, Rizzuto DS, Kahana MJ, Lega BC (2017) Theta band power increases in the posterior hippocampus predict successful episodic memory encoding in humans. Hippocampus 27:1040-1053.

Ludbrook J (1994) Advantages of permutation (randomization) tests in clini$\mathrm{cal}$ and experimental pharmacology and physiology. Clin Exp Pharmacol Physiol 21:673-686.

Mormann F, Fell J, Axmacher N, Weber B, Lehnertz K, Elger CE, Fernández G (2005) Phase/amplitude reset and theta-gamma interaction in the human medial temporal lobe during a continuous word recognition memory task. Hippocampus 15:890-900.

Nelson DL, McEvoy CL, Schreiber TA (2004) The University of South Florida free association, rhyme, and word fragment norms. Behav Res Methods Instrum Comput 36:402-407.

Pacheco Estefan D, Sánchez-Fibla M, Duff A, Principe A, Rocamora R, Zhang H, Axmacher N, Verschure P (2019) Coordinated representational reinstatement in the human hippocampus and lateral temporal cortex during episodic memory retrieval. Nat Commun 10:2255.

Poppenk J, Evensmoen HR, Moscovitch M, Nadel L (2013) Long-axis specialization of the human hippocampus. Trends Cogn Sci 17:230-240.

Ritchey M, Libby LA, Ranganath C (2015) Cortico-hippocampal systems involved in memory and cognition: the PMAT framework. Prog Brain Res 219:45-64.

Rizzuto DS, Madsen JR, Bromfield EB, Schulze-Bonhage A, Seelig D, Aschenbrenner-Scheibe R, Kahana MJ (2003) Reset of human neocortical oscillations during a working memory task. Proc Natl Acad Sci U S A 100:7931-7936.

Rizzuto DS, Madsen JR, Bromfield EB, Schulze-Bonhage A, Kahana MJ (2006) Human neocortical oscillations exhibit theta phase differences between encoding and retrieval. Neuroimage 31:1352-1358.

Rugg MD, Johnson JD, Park H, Uncapher MR (2008) Encoding-retrieval overlap in human episodic memory: a functional neuroimaging perspective. Prog Brain Res 169:339-352.
Seltman H (2009) Mixed models. In: Experimental design and analysis, pp 357-377.

Staresina BP, Michelmann S, Bonnefond M, Jensen O, Axmacher N, Fell J (2016) Hippocampal pattern completion is linked to gamma power increases and alpha power decreases during recollection. Elife 5: e17397.

Staudigl T, Hanslmayr S (2013) Theta oscillations at encoding mediate the context-dependent nature of human episodic memory. Curr Biol 23:1101-1106.

Stella F, Treves A (2011) Associative memory storage and retrieval: involvement of theta oscillations in hippocampal information processing. Neural Plast 2011:683961

Strehl A, Ghosh J, Mooney R (2000) Impact of similarity measures on webpage clustering. Paper presented at Seventeenth National Conference on Artificial Intelligence (AAAI 2000), Austin, TX, July.

Thakral PP, Wang TH, Rugg MD (2015) Cortical reinstatement and the confidence and accuracy of source memory. Neuroimage 109:118129.

Van Dongen S, Enright AJ (2012) Metric distances derived from cosine similarity and Pearson and Spearman correlations. arXiv:1208. $3145 \mathrm{v} 1$.

Watrous AJ, Miller J, Qasim SE, Fried I, Jacobs J (2018) Phase-tuned neuronal firing encodes human contextual representations for navigational goals. Elife 7:e32554.

Xia M, Wang J, He Y (2013) BrainNet Viewer: a network visualization tool for human brain connectomics. PLoS One 8:e68910.

Yaffe RB, Kerr MS, Damera S, Sarma SV, Inati SK, Zaghloul KA (2014) Reinstatement of distributed cortical oscillations occurs with precise spatiotemporal dynamics during successful memory retrieval. Proc Natl Acad Sci U S A 111:18727-18732.

Yamaguchi Y, Sato N, Wagatsuma H, Wu Z, Molter C, Aota Y (2007) A unified view of theta-phase coding in the entorhinal-hippocampal system. Curr Opin Neurobiol 17:197-204. 